\title{
Electrophysiological Evidence of Intuition: Part 2. A System-Wide Process?
}

\author{
ROLLIN McCRATY, Ph.D., ${ }^{1}$ MIKE ATKINSON, ${ }^{1}$ and RAYMOND TREVOR BRADLEY, Ph.D. ${ }^{2}$
}

\begin{abstract}
Objectives: This study aims to contribute to a scientific understanding of intuition, a process by which information normally outside the range of conscious awareness is perceived by the body's psychophysiological systems. The first objective, presented in two empirical reports (Part 1 and Part 2), was to replicate and extend the results of previous experiments demonstrating that the body can respond to an emotionally arousing stimulus seconds before it is actually experienced. The second objective, to be presented in a forthcoming publication (Part 3), is to develop a theory that explains how the body receives and processes information involved in intuitive perception.

Design: The study used a counterbalanced crossover design, in which 30 calm and 15 emotionally arousing pictures were presented to 26 participants under two experimental conditions: a baseline condition of "normal" psychophysiologic function and a condition of physiological coherence. Primary measures included: skin conductance; the electroencephalogram (EEG), from which cortical event-related potentials (ERP) and heartbeatevoked potentials (HBEP) were derived; and the electrocardiogram (ECG), from which cardiac decelerations/ accelerations were derived. These measures were used to investigate where and when in the brain and body intuitive information is processed.

Results: The main findings presented here are: (1) surprisingly, both the heart and brain appear to receive and respond to intuitive information; (2) even more surprisingly, there is compelling evidence that the heart appears to receive intuitive information before the brain; (3) there were significant differences in prestimulus ERPs for calm versus emotional stimuli; (4) the frontal cortex, temporal, occipital, and parietal areas appear to be involved in the processing of prestimulus information; (5) there were significant differences in prestimulus calm/emotional HBEPs, primarily in the coherent mode; (6) there were significant gender differences in the processing of prestimulus information. Especially noteworthy is the apparent interaction between the HBEPs and ERPs in the females, which suggests that the heart modulates the ERP and that females are more attuned to intuitive information from the heart.

Conclusions: Overall, our data suggest that the heart and brain, together, are involved in receiving, processing, and decoding intuitive information. On the basis of these results and those of other research, it would thus appear that intuitive perception is a system-wide process in which both the heart and brain (and possibly other bodily systems) play a critical role. To account for the study's results, Part 3 will develop a theory based on holographic principles explaining how intuitive perception accesses a field of energy into which information about "future" events is spectrally enfolded.
\end{abstract}

\footnotetext{
${ }^{1}$ HeartMath Research Center, Institute of HeartMath, Boulder Creek, CA.

${ }^{2}$ Institute for Whole Social Science, Carmel, CA.
} 


\section{INTRODUCTION}

$\mathbf{A}$ mysterious yet familiar facet of experience, intuition is a powerful transforming psychosocial agent that not only reshapes the direction of individual lives but also often changes the course of human history. Intuitive perception is commonly acknowledged to play an important role in business, learning, creativity, medical diagnosis, healing, spiritual growth, and overall well-being (Myers, 2002). Yet despite its subtle presence in informing such diverse aspects of individual and collective life, intuition is poorly understood and remains a scientific enigma.

Our major objective in the research reported here is to contribute to a scientific understanding of intuition-of how information beyond normal conscious experience is perceived by the body's psychophysiologic systems. Along with other scientists who deem these phenomena as worthy of rigorous investigation, we believe that such intuitive perception involves the body's connection to a field of information beyond normal conscious awareness (Bohm, 1980; Bradley, 1987; Laszlo, 1995; Loye, 1983; Nadeau and Kafatos, 1999; Pribram, 1991; Sheldrake, 1999).

We take an information processing perspective, and regard intuition as a process by which information normally outside the range of cognitive processes is sensed and perceived in the body and mind as certainty of knowledge or feeling (positive or negative) about the totality of a thing distant or yet to happen (McCraty et al., 2004). This "thing" can be a material object or event, or a mental construct such as a thought or idea. Overall, the intuitive experience of an immediate, total sense of the thing as a whole is quite unlike the incremental experience of normal awareness in which the mind's contents are updated sequentially as sensory experience unfolds. For our research purposes, it is important to note that the experience of intuition is not confined to cognitive-based perception, but involves the entire psychophysiologic system, often manifesting through a wide range of emotional feelings and physiologic changes experienced throughout the body.

The capacity to process information about distant events appears to be a property of all physical and biologic organization, and is likely due to nonlocal communication via coherent oscillations in the energy field that interconnects everything in the universe (Bohm and Hiley, 1993; Laszlo, 1995; Nadeau and Kafatos, 1999). Thus, at the subatomic level, physicists have shown in such famous experiments as the "double-slit" experiment that subatomic particles (photons) behave as if they "know" the outcome of future events before these events actually happen (Aczel, 2002; Penrose, 1989). At the elementary level biologic organization, cellular structures appear to have immediate knowledge of remote actions in the system, thereby enabling the emergence of spontaneous long-range cooperative organization in biomolecules and membranes (Hameroff, 1987), in dendritic networks of cortical neurons (Shepherd et al, 1985), and in colonies of single-celled organisms such as bacteria (BenJacob et al., 2000). At the level of multicellular organisms, a prestimulus response has been experimentally demonstrated in earthworms.* At higher levels of life, accurate premonitions of natural disasters by animals have been documented throughout recorded history (Sheldrake et al., 1998), and there is recent experimental evidence that pets appear to "know" the moment their owners decide to return home, despite being separated by many miles (Sheldrake, 1999).

In short, this capacity for processing information about future and nonlocal events is an ability that is not peculiar to humans, and neither is it a capacity solely of the brain alone. Moreover, recent prior research on humans has found that the autonomic nervous system exhibits a prestimulus response, reflected in changes in skin conductance (Radin, 1997b; Spottiswoode and May, 2003), and in Part 1 of this study we reported evidence showing that the heart responds to prestimulus information about a future event seconds before it is actually experienced (McCraty et al., 2004).

In light of these considerations, therefore, the central proposition of our research is that intuitive perception is a system-wide process in which the heart and brain and other bodily systems apprehend and process prestimulus information about a future event. In the same way that the findings in physics of such space/time-defying nonlocal communication ultimately revolutionized scientific understanding of the physical world, we believe that rigorous investigation of intuitive perception is of equal scientific importance. This is because the results of such work have the potential not only to yield a new understanding of human perception and consciousness, but also to transform the way we view our abilities and our relations to the world.

This is the second report on the results of a study on intuition in which we investigated, under controlled experimental conditions, the heart and brain's response to information about a future event before the event occurs. ${ }^{\dagger}$ In the first report (McCraty et al., 2004), we presented surprising evidence that the heart appears to receive and respond to intuitive information. Specifically, participants demonstrated a significantly greater heart rate deceleration prior to presentation of future emotional stimuli compared to calm stimuli. Furthermore, the shape of the deceleration curves provide evidence that the psychophysiologic system responds to an unknown stimulus in the same way it responds to a known future stimulus, suggesting that intuitive information is processed similarly to conventional sensory input.

Here we present the remainder of our results, which show where in the brain intuitive information is processed and the

*Wildey CR. Biological Response to Stimulus [Master's thesis], Electrical Engineering, University of Texas at Arlington, December 2001 .

${ }^{\dagger}$ It is important to note, as made explicit in Part 1, that our study investigated only the temporal dimension of intuition - the proposition that the body receives and processes information about a future event before the event actually happens. 
temporal dynamics of the communication of intuitive information between the heart and brain. Finally, Part 3 of this work (forthcoming) will develop a theory, based on the principles of holographic organization, to explain intuitive perception by describing how the body is connected, via sensory perception, to a field of energy that spectrally enfolds information about "future" events.

\section{Previous research}

The concept that intuitive perception is purely a function of the unconscious mind accessing forgotten prior experience has been challenged by several recent studies. Using rigorous experimental protocols, these studies have shown that the body often responds to a future emotionally arousing stimulus 4 to 7 seconds prior to experiencing the stimulus (Bierman, 2000; Radin, 1997b, 2004; Spottiswoode and May, 2003).

The first research we are aware of to examine changes in brain activity that preceded an unknown stimulus was conducted by Levin and Kennedy (1975). They observed a significantly larger contingent negative variation (a slow brain wave potential associated with anticipation, expectancy, or cortical priming) just before subjects responded to a target stimulus presented at random.

Later, Warren et al. (1992a, 1992b) also found significant differences in event-related potentials (ERP) ${ }^{\ddagger}$ between target and nontarget stimuli presented during forced-choice precognition tasks. Don and colleagues extended these ERP findings in a series of gambling studies, which demonstrated enhanced negativity widely distributed across the scalp in response to future targets (Don et al., 1998; McDonough et al., 2002). These researchers concluded that the ERP effect was an indicator of "unconscious precognition" based on their findings that although a transfer of information about the future event had been measured physiologically, this information did not appear to reach conscious awareness (McDonough et al., 2002). $\S$

Recently, researchers have explored physiologic predictors of future events by investigating whether the human autonomic nervous system can unconsciously respond to randomly selected future emotional stimuli. Radin (1997a, 1997b) designed experiments to evoke an emotional response using randomly selected emotionally arousing or calming photographs. Indicators of autonomic activity included skin conductance level (SCL) and photoplethysmographic mea-

${ }_{\ddagger}^{\ddagger}$ Event-related potentials are voltage fluctuations that are associated in time with some physical, mental, or emotional occurrence. These potentials can be recorded from the scalp and extracted from the ongoing electroencephalogram (EEG) by means of filtering and signal averaging.

${ }^{\S}$ Although not investigating prediction of future events, a study of Pavlovian conditioning also found a significant ERP difference in the prestimulus phase of an experiment, before subjects had any way of knowing which of two stimuli would be later presented (Paige et al., 1987). sures of heart rate and blood volume. Comparison of SCL response between emotional and calm trials showed a significantly greater change in electrodermal activity around $5 \mathrm{sec}-$ onds before a future emotional picture than before a future calm picture. These results have since been replicated (Bierman, 2000; Bierman and Radin, 1997; Bierman and Scholte, 2002; Radin, 2004). ., , A further study, using a free-running protocol, ${ }^{\#}$ also found significant skin conductance changes in the prestimulus period (Spottiswoode and May, 2003).

Extending Radin's approach, in Part 1 of this study (McCraty et al., 2004), we reported a significantly greater heart rate deceleration prior to presentation of future emotional stimuli compared to calm stimuli. Consistent with cultural views of gender differences in intuitive aptitude, we also found that females were more responsive to prestimulus information than males. We offer further findings on gender differences in this report.

In light of evidence of intuitive-like phenomena at diverse levels of biologic organization (see Introduction) and our findings in Part 1, it is likely that the processing of prestimulus information about a future event is not confined to the brain alone. Instead, it is hypothesized that intuitive perception is a system-wide process in which the heart and brain (and possibly other bodily systems) are involved together in responding to intuitive information.

\section{RESEARCH DESIGN AND METHODS}

We adopted Radin's (1997b) basic experimental protocol (Fig. 1) while including additional measures of brain and heart activity well-suited to investigate information processing. This was done to determine where and when in the brain and body information about the future event was registered and processed. In addition to SCL, we included the electrocardiogram (ECG) for measurement of heart rate variability (beat-to-beat decelerations/accelerations) and a 19channel electroencephalogram (EEG) for cortical ERP and heartbeat-evoked potential measurements. These measures have all been used to index specific aspects of sensory information processing, and can be interpreted according to well-established operational criteria (see Discussion).

This study utilized a counterbalanced crossover design with two experimental conditions.** Each research subject

"Bem DJ. Precognitive habituation: Replicable evidence for a process of anomalous cognition. Unpublished manuscript, 2003.

IRadin DI. Evidence for an anomalous anticipatory effect in the autonomic nervous system. Unpublished manuscript, 2002.

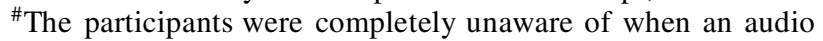
startle stimulus would be randomly presented.

${ }^{* *}$ The counterbalanced design was necessary to control for exposure effects. Half the participants completed the experimental protocol in condition 1 first, whereas the other half completed the protocol in condition 2 first. The two testing sessions were spaced 2 weeks apart, with the order of the two experimental conditions randomly assigned for each participant. 

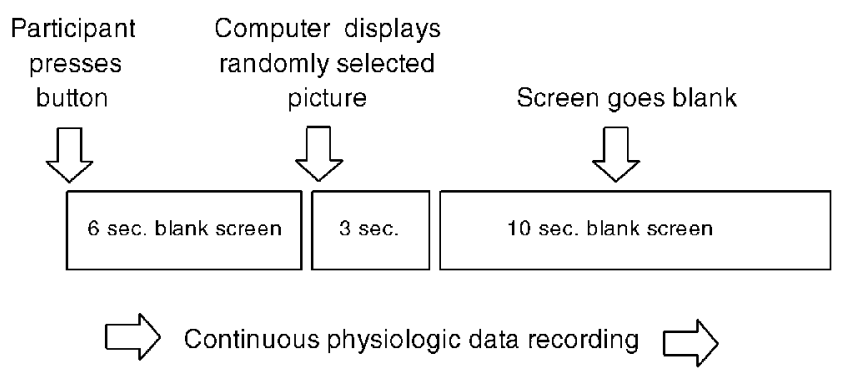

FIG. 1. Experimental protocol. Participants viewed a computer monitor and were instructed to press a button when ready to begin each trial. Following the button press, the monitor remained blank for 6 seconds, after which the computer presented a randomly selected image from one of the two picture sets (calm or emotional) and displayed it for 3 seconds. A blank screen followed for 10 seconds. After this cool-down period, a message appeared on the monitor, instructing participants to begin the next trial when ready.

participated in the protocol twice: once in his or her baseline psychophysiologic state (condition 1), and once after having maintained the physiological coherence mode ${ }^{\dagger \dagger}$ for 15 minutes prior to participation in the session (condition 2). ${ }^{\ddagger}$ The post-physiological coherence condition was included to test the hypothesis that an enhanced prestimulus response is related to the maintenance of a state of physiological coherence. We have previously found that increased heart rhythm coherence correlates with significant improvements in performance on tasks requiring attentional focus and subtle discrimination (McCraty, 2002; McCraty and Atkinson, 2003), which may be important elements of the intuitive effect studied here.

Each participant viewed 45 pictures in each of the two

\footnotetext{
${ }^{\dagger}$ As documented elsewhere, we previously introduced the term physiological coherence to describe a specific physiologic mode frequently associated with sustained positive emotions. This mode encompasses distinct but related physiologic phenomena including entrainment, resonance, and synchronization, which reflect more efficient and harmonious interactions among the body's subsystems (McCraty and Childre 2002, 2004; Tiller et al., 1996). Correlates of physiological coherence include: increased synchronization between the two branches of the autonomic nervous system, a shift in autonomic balance toward increased parasympathetic activity, increased heart-brain synchronization, increased vascular resonance, and entrainment between diverse physiological oscillatory systems. The coherent mode is reflected by a smooth, sine wave-like pattern in the heart rhythms and a narrow-band, highamplitude peak in the low-frequency range of the heart rate variability power spectrum, at a frequency of approximately 0.1 hertz (Tiller et al., 1996).

¥To induce the physiological coherence mode, participants practiced the Heart Lock-In emotional restructuring technique for 15 minutes before beginning the session. Combining intentional heart focus with the self-generation of a genuine positive emotion such as appreciation or care, the Heart Lock-In technique has previously been shown to induce development and maintenance of the physiological coherence mode (for details of this technique, see Childre and Martin, 1999; McCraty and Childre, 2002).
}

experimental sessions; each session consisted of $30 \mathrm{calm}$ pictures and 15 emotional pictures selected from the International Affective Picture System (IAPS). This 2:1 ratio was used to avoid physiologic habituation to the emotional pictures (Boucsein, 1992). II Data from a total of 2340 trials were collected across the two sessions for all participants.

Details of the testing procedure, stimulus selection and presentation, and SCL and ECG measurement are provided in Part 1 (McCraty et al., 2004).

\section{Participants}

Twenty-six (26) adult participants, 11 males, 15 females, ranging in ages from $28-56$ (mean age, 45 ), were recruited from e-mail notices to people who had prior training in the HeartMath emotional management techniques. ${ }^{\S}$ All participants were in good health and had normal or corrected-tonormal vision, and all gave informed consent.

\section{Measures}

Each participant was fitted with an EEG electrode cap (19 channels) according to the International 10-20 System. Conducting gel (Electro-Cap International, Inc., Eaton, OH) was used to ensure that all electrode impedances were below $5 \mathrm{k} \Omega$. The reference was linked mastoids. An additional electrode for recording the electrooculogram (EOG) was placed above the right eye to monitor eye blinks and movement. The EEG signals were amplified with a Grass model 8-18D electroencephalograph system.

\section{Data and statistical analysis}

Data editing was blind to stimulus category (calm or emotional targets). Data processing and statistical analysis used DADISP 4.1 (DSP Development Corporation, Newton, MA), MATLAB 6.1 (The MathWorks, Inc., Natick, MA), and SPSS 8.0 (SPSS, Inc., Chicago, IL) software.

To reduce the possibility of false-positive findings, we chose statistically conservative procedures for data analysis. Because it controls for autocorrelations inherent in physiologic signals and their underlying non-normal distributions, randomized permutation analysis (RPA; Blair and Karniski, 1993) was used to determine statistical significance of the differences between emotional and calm curves during the prestimulus period. For a description of the operational procedures for RPA see Part 1 (McCraty et al., 2004).

ECG data. The ECG data used for heart rate variability (HRV) and heartbeat-evoked potential analysis were all nor-

$\S \S$ The HeartMath techniques are a set of positive emotion-refocusing and emotional restructuring interventions designed to facilitate stress reduction, promote health, and enhance performance (Childre and Martin, 1999). A number of studies have documented that these techniques facilitate the self-generation and maintenance of the physiological coherence mode (McCraty and Childre, 2002). 
mal sinus intervals. All aberrant beats and artifacts were removed from the records: a computer algorithm eliminated intervals that varied by more than $30 \%$ of the mean of the previous 4 intervals, and any remaining artifacts were removed during second-stage editing by an experienced technician who visually inspected the records. A regularly spaced time series was derived from the succession of normal RR intervals by linear interpolation of the irregularly spaced series and then resampled at 8 samples per second.

EEG data. To decrease processing time for the 44,460 segments (2340 trials $\times 19$ channels) of EEG data, all channels were filtered using a $30-\mathrm{Hz}$ low-pass digital filter and resampled at 128 samples per second. Next, each of the EEG channels and the EOG channel were raveled into 19-secondlong single-trial epochs, where trial initiation ("button press") marked the start of the epoch. Independent component analysis $\|$ | was used to remove eye blink and muscle artifacts from the data, with manual verification to ensure data integrity. Trials containing artifacts were not used in statistical analysis.

Event-related potentials. ERPs were calculated to enhance the underlying electrical EEG waveforms at each site. Taking a conservative approach, we removed high-frequency components from each trial's ERP, filtering it with a Butterworth low-pass filter with a $1-\mathrm{Hz}$ cutoff and resampling at 8 samples per second. ${ }^{\text {III }}$ All filtered artifact-free trial ERP waveforms from each session were averaged, constructing one calm and one emotional session ERP for each participant's session. The prestimulus data (48 points) were extracted and subjected to a randomization test (Blair and Karniski, 1993), which is a rigorous method for identifying statistically significant waveform difference potentials.

Briefly, each participant's calm and emotional session ERP waveform pairs were tested with a point-by-point paired-samples $t$ statistic, by pairing each participant's emotional ERP data points with his or her respective calm ERP data points. This yields $48 t$ statistics-one for each time point. Two multivariate statistics were computed. The first, $t_{\text {sum }}$, is sensitive to an overall difference between the calm and emotional waveforms, and was computed by summing all 48 univariate $t$ statistics. The second statistic, $t_{\max }$, is the greatest absolute value of $t$ in the 48-t-statistic series. Because $t_{\max }$ is sensitive to a change in only one of the variables and to effects of short duration, it indicates where in time differences occur between the calm and emotional waveforms.

Finally, a reference distribution was constructed by ran-

|| $\|$ Scott Makeig, Computational Neurobiology Laboratory, Salk Institute, La Jolla, CA.

IIIT The question of whether significant information is actually contained in the high-frequencycomponents should be investigated in future studies. domly reassigning (within subjects) the calm/emotional ERP pair designations and recalculating $t_{\text {sum }}$ and $t_{\max }$ from the new assignment. A rank-ordered reference distribution of 10,000 random permutations was the result, which meant that the observed statistic could be referenced to this distribution to obtain a $p$ value (Blair and Karniski, 1993).

Separate randomization tests were performed for each EEG channel by experimental condition and gender.

Heartbeat-evoked potentials. To examine changes in cardiac-related afferent information processing during the prestimulus period, heartbeat-evoked potential (HBEP) analysis was performed. ${ }^{\# \#}$ First we extracted a 700-ms-long segment of data from each EEG channel that was time-locked to each of the first 4 ECG cycles occurring immediately after each trial's initiation (button press). These 4 EEG segments were averaged to form the trial's HBEP waveform. The 700-ms-long EEG segments began $100 \mathrm{~ms}$ before the $\mathrm{R}$-wave and continued $600 \mathrm{~ms}$ after the $\mathrm{R}$-wave. To remove the high-frequency EEG components, each trial's HBEP was filtered using a Butterworth low-pass filter with a 1-Hz cutoff. All filtered trial HBEP waveforms were averaged to form one calm and one emotional session HBEP for each participant. These HBEPs were then subjected to the randomization test described above (see Event-related potentials section). Separate randomization tests were performed for each EEG channel by experimental condition and gender.

\section{RESULTS}

In the first report from this study on physiologic responses to future stimuli (McCraty et al., 2004), we presented data showing that the heart appears to receive and respond to intuitive information, as summarized above. Here, in presenting the remaining results from our study, we include data on the brain's response to the prestimulus information and data on the interaction between the heart and brain in receiving and processing prestimulus information.

\section{Univariate analysis}

Event-related potentials. Results for the group as a whole in condition 1 showed a significant $t_{\text {sum }}$ difference in ERPs in the prestimulus period for future calm versus emotional photos at both frontopolar EEG sites, FP1 (left frontopolar; $t_{\text {sum }}=-28.82, p<0.05$ ) and FP2 (right frontopolar; $t_{\text {sum }}=$ $-27.27, p<0.05$ ), indicating a difference in the overall waveforms at these locations (Fig. 2). The ERPs for a fu-

\footnotetext{
\#\#When signal averaging techniques are used to trace the flow and processing of afferent neurological signals from the heart through the different regions of the brain, the resulting waveforms are called heartbeat-evoked potentials. The peak of the ECG Rwave is used as the timing source for the signal averaging process.
} 


\section{Condition 1-ERP : All Subjects}
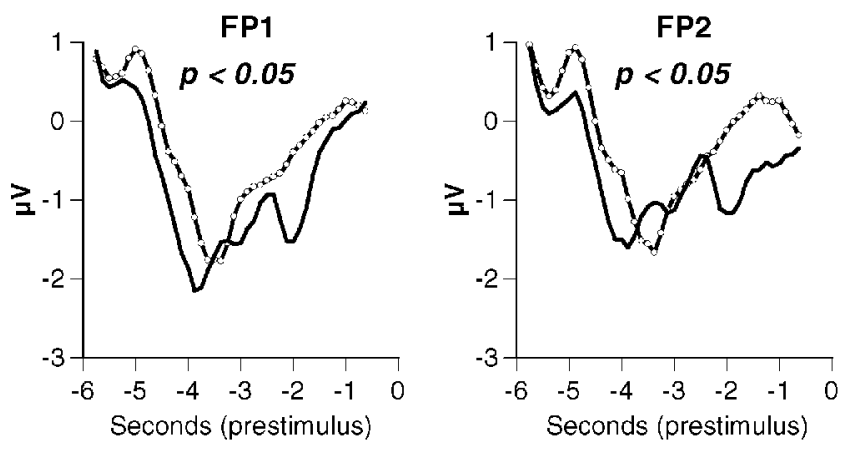

- Calm

FIG. 2. Mean event-related potential (ERP) waveforms at FP1 (left frontal cortex) and FP2 (right frontal cortex) for the group as a whole $(n=26)$ for calm versus emotional trials. Data are shown for the 6-second prestimulus period for condition 1 (with the " 0 " time point denoting stimulus onset). There were significant differences in the overall waveforms at these sites.

ture emotional stimulus were more negative, with the point of maximum negativity occurring slightly before that of the ERPs for the future calm pictures. There was also a positive shift with a steep slope observed approximately 4 seconds before the stimulus. In both locations this positive shift in the emotional trial ERP occurred around a second before the shift occurred in the calm trial ERPs. In condition 2, there was a significant $t_{\text {sum }}$ difference between the prestimulus ERPs for calm versus emotional trials at midline EEG site $\mathrm{Pz}\left(t_{\text {sum }}=-13.24, p<0.05\right)$. Because of the significant findings at FP1 and FP2, an additional RPA of the EOG channel was conducted, which revealed that eye movement artifacts did not contribute to this result.

Heartbeat-evoked potentials. No significant differences were observed in the prestimulus HBEPs for the group as a whole.

\section{Bivariate analysis}

The ERP and HBEP data were also analyzed by experimental condition and gender.

Event-related potentials. The results for the ERPs in condition 1 for the female subgroup showed a significant $t_{\text {sum }}$ prestimulus difference between the calm and emotional trials at FP2 $\left(t_{\text {sum }}=-30.40, p<0.05\right)$ and a marginally significant difference at FP1 $\left(t_{\text {sum }}=-30.05, p=0.06\right)$, indicating a difference in the overall waveforms at these frontal locations (Fig. 3). Again, the ERPs for future emotional stimuli were more negative than the calm trial ERPs, and the positive shift in the emotional trial ERPs occurred prior to the positive shift in the calm trial ERPs.

In condition 2, the females demonstrated significant $t_{\max }$ differences in the ERP waveforms at O1 (left occipital; $t_{\max }=3.81, p<0.05$ ) and T5 (left posterior temporal; $\left.t_{\max }=4.59, p<0.05\right)$, around 0.75 seconds prior to the stimulus.

In the male subgroup for condition 1, there were significant differences between calm and emotional ERPs at $\mathrm{O} 2$ (right occipital; $\left.t_{\text {sum }}=-32.66, p<0.05\right)$, T5 $\left(t_{\max }=4.10, p<0.05\right)$, and FP1 $\left(t_{\max }=3.21, p<0.05\right)$. In condition 2 (coherence) there were significant differences at $\mathrm{T} 5\left(t_{\max }=4.58, p<0.05\right)$, P3 (left parietal; $t_{\text {sum }}=-22.67, p<0.01 ; t_{\text {max }}=7.28, p<$ $0.01)$, and both $\mathrm{O} 1\left(t_{\max }=6.42, p<0.05\right)$ and $\mathrm{O} 2\left(t_{\max }=\right.$ $4.07, p<0.05$ ), all of which occurred approximately 4 seconds prior to the stimulus. There were also significant differences in the overall waveforms at both occipital areas, O1 $\left(t_{\text {sum }}=\right.$ $-24.41, p<0.01)$ and $\mathrm{O} 2\left(t_{\text {sum }}=-18.85, p<0.01\right)$; the ERPs for the emotional trials were more negative.

Heartbeat-evoked potentials. RPA of the heartbeatevoked potential data revealed a number of significant findings. In condition 1 for the females, there was a significant difference between the HBEP waveforms for the calm and emotional trials at the right lateral frontal site F8 $\left(t_{\text {sum }}=\right.$ $-16.76, p<0.05)$. The waveform for trials with a future

\section{A Condition 1-ERP : Female Subgroup}
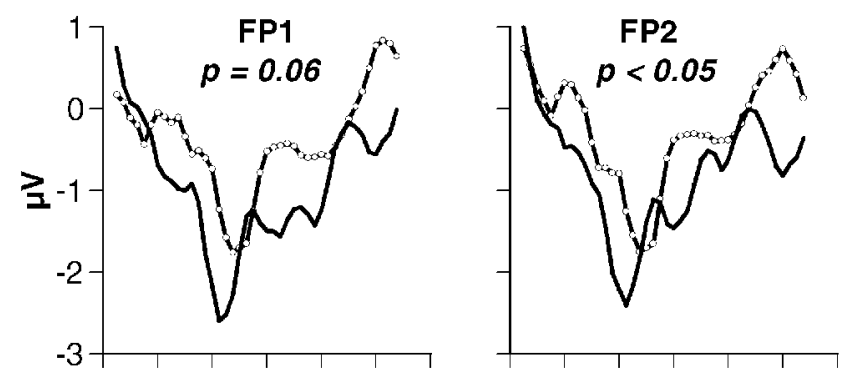

\section{B Condition 2-ERP : Female Subgroup}
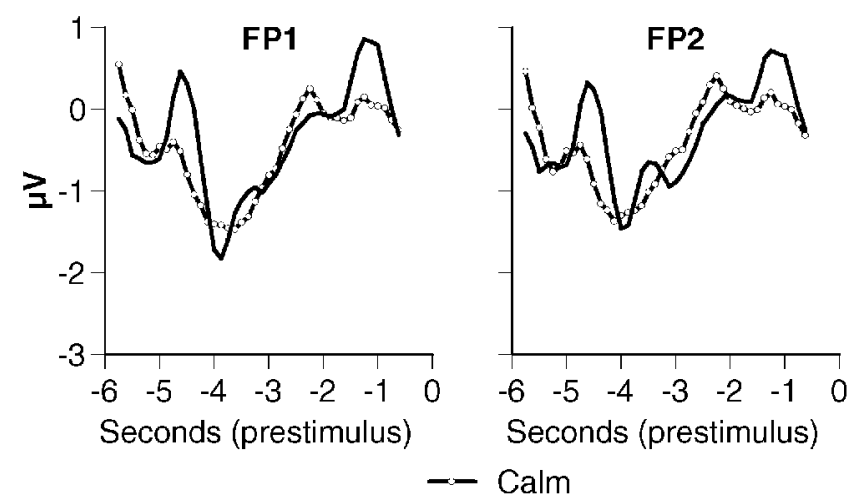

FIG. 3. Mean event-related potential (ERP) waveforms at FP1 and FP2 for the female subgroup $(n=15)$ for calm versus emotional trials. Data are shown for the prestimulus period for condition 1 (A) and condition 2 (B) (with the "0" time point denoting stimulus onset). In condition 1 , there was a marginally significant difference in the overall waveforms for calm versus emotional trials at FP1 and a significant difference at FP2. 
emotional stimulus had a higher positive level for the first $100 \mathrm{~ms}$ after button press and then quickly declined, maintaining greater negativity for the remaining time period; although a positive shift in both waveforms was observed 200 $\mathrm{ms}$ later. The first 100-ms region corresponds to the pre-Rwave portion of the HBEP. This same trend is evident at most of the EEG sites, especially the frontal locations.

In condition 2 for the female subgroup, there were significant differences in the calm and emotional HBEP waveforms at both frontopolar locations, FP1 $\left(t_{\text {sum }}=9.73, p<0.05\right)$ and FP2 $\left(t_{\text {sum }}=9.67, p<0.05\right)$ (Fig. 4$)$. In condition 2 , the waveforms have a markedly different morphology than in condition 1: as shown in Figure 4, the emotional waveform starts more negative than the calm waveform and crosses over the calm waveform much earlier (approximately 120 seconds postbutton press), remaining more positive before sloping back negatively to cross the calm trial waveform again ap-

$\mathbf{A}$

Condition 1-HBEP: Female Subgroup
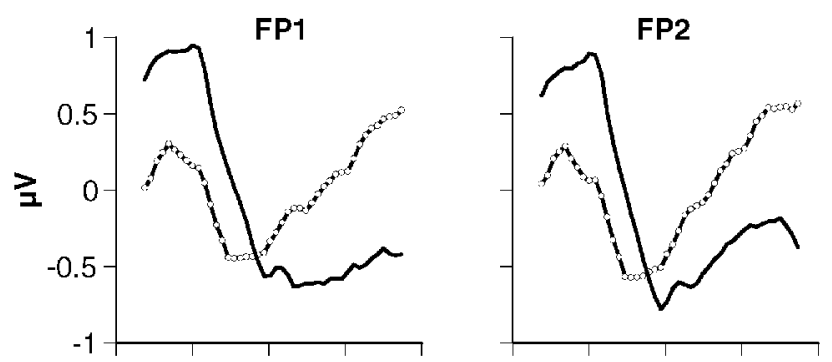

B
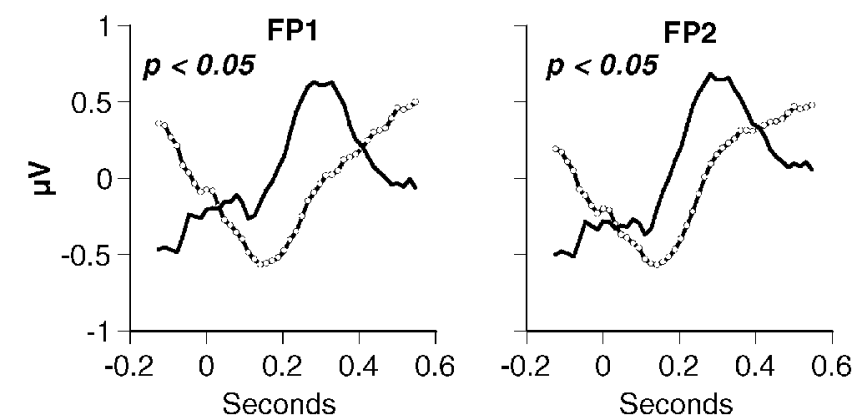

-- Calm

- Emotional

FIG. 4. Mean prestimulus heartbeat-evoked potential (HBEP) waveforms at FP1 and FP2 for the female subgroup $(n=15)$ for calm versus emotional trials. Data are shown for condition 1 (A) (baseline psychophysiologic mode) and condition 2 (B) (postphysiologicalcoherence), with the " 0 " time point denoting the peak of the ECG R-wave. In condition 2, there were significant differences in the HBEP waveforms for calm versus emotional trials at both frontal sites. Note the marked differences in the morphology of the waveforms in condition 2 compared to condition 1 . It is noteworthy that the ERP analysis for the female subgroup revealed significant differences at FP1 and FP2 in condition 1, but not in condition 2 (see Fig. 3), which suggests that information processing in the frontal cortex was modified by afferent input from the heart. proximately $400 \mathrm{~ms}$ later. There was also a significant difference at left anterior temporal site T3 $\left(t_{\text {sum }}=11.19, p<\right.$ $0.05)$. This pattern of activity was similar to that observed for FP1 and FP2. In summary, in condition 2, the HBEP differences were more pronounced in the frontal areas and in the left hemisphere, and the morphology of the waveforms was inverted relative to that found in condition 1 .

While no significant differences in HBEPs were observed for the males in experimental condition 1, there was a significant difference at right parietal site $\mathrm{P} 4\left(t_{\max }=1.19, p<\right.$ $0.05)$ in condition 2.

In reviewing the statistical results, it should be noted that: our significant findings are consistent with those of other studies conducted on independent samples; our participants went through the experimental sessions twice and produced the same pattern of results; and we also analyzed our data using an alternative statistical procedure (principal components analysis) and found essentially the same pattern of results (not presented here). Thus, it is most unlikely that multiple-comparison errors contaminate our major results.

\section{DISCUSSION}

This study's purpose was to independently replicate and extend previous experiments demonstrating physiologic responses to an emotional stimulus prior to its presentation. While confirming this finding overall, we were unable to replicate the skin conductance results, as reported in Part 1 (McCraty et al., 2004).***

Our working assumption is that no matter how intuitive information is initially introduced into the psychophysiologic systems, once received it is processed in the same way as information obtained through the familiar sensory systems. This perspective is supported by the HRV data reported in Part 1. These data indicate that, on average, the informational input to the heart regarding the future emotional stimulus occurred approximately 4.75 seconds before the stimulus was actually presented. This is where the slope of the heart rate deceleration curve for the emotional trials clearly starts to diverge from the slope for the calm trials (Fig. 5). In addition, based on classical interpretations of cardiac decelerations/accelerations in relation to the processing of sensory information, our data suggest that the heart responds to the unknown stimulus in the same way it does when the future stimulus is known (McCraty et al., 2004).

\section{Event-related potentials}

A number of components reflecting various aspects of information processing have been described in the ERP liter-

${ }^{* * *}$ This difference in SCL results appears mainly due to sampling differences between our respective studies (see Part 1 for details). 
ature. A large body of research has investigated the phenomenon of "mismatch negativity," where a larger negative signal is elicited in response to a novel or infrequent stimulus (Naatanen et al., 1993; Tervaniemi et al., 1994). Pribram (1991) suggests that the initial negative-going ERP wave can generally be interpreted as representing attentional processes - that is, the brain says to itself: "I have to pay attention, stay alert, and process this information," with a positive shift representing the end of processing: "The information is here, I have to do something," and an additional negative shift representing an updating and reevaluation process.

Increased negativity in the emotional trial ERPs was clearly observed in the females, who also had a significant heart rate deceleration and significantly different heartbeatevoked potentials. In condition 1 (baseline psychophysiologic mode), the females' ERPs for the emotional trials had greater negativity, which was maintained throughout the prestimulus period. However, there was a sharp positive shift approximately 3.5 seconds prior to stimulus presentation, which leveled off and then shifted positive once again in both frontal hemispheres. If Pribram's interpretation is correct, it would suggest that the observed positive shift in the ERPs in our data was an important indicator of when the brain "knew" the nature of the future stimulus.

Overall, our ERP data are consistent with previous research showing that the frontal areas are involved in the processing of intuitive or precognitive information (Don et al., 1998; Warren et al., 1992a, 1992b). Moreover, our data also indicate the involvement of the temporal, occipital, and parietal areas.

\section{Heartbeat-evoked potentials}

When signal averaging is used to trace afferent neural signals from the heart, the peak of the ECG R-wave is used as the timing source for event-related activity in the brain, and the resulting waveform is called a heartbeat-evoked potential (HBEP). The potentials in the HBEP waveform reflect the processing of cardiovascular-related afferent signals occurring in different areas of the brain (Schandry and Montoya, 1996).

It has been demonstrated that afferent input from the heart evokes cortical responses analogous to "classical" sensory event-related potentials (Schandry and Montoya, 1996; Schandry et al., 1986). These experiments have shown that afferent input from the cardiovascular system is coupled with specific changes in the brain's electrical activity, most pronounced at the frontocortical areas-a region particularly involved in processing visceral afferent information. This suggests that perceptual processing of internal events by the central nervous system is comparable to perceptual processing of external events, and that the effects of cardiovascular input are very specific. Thus, the influence of the heart on the brain is not diffuse, but discrete, resulting in

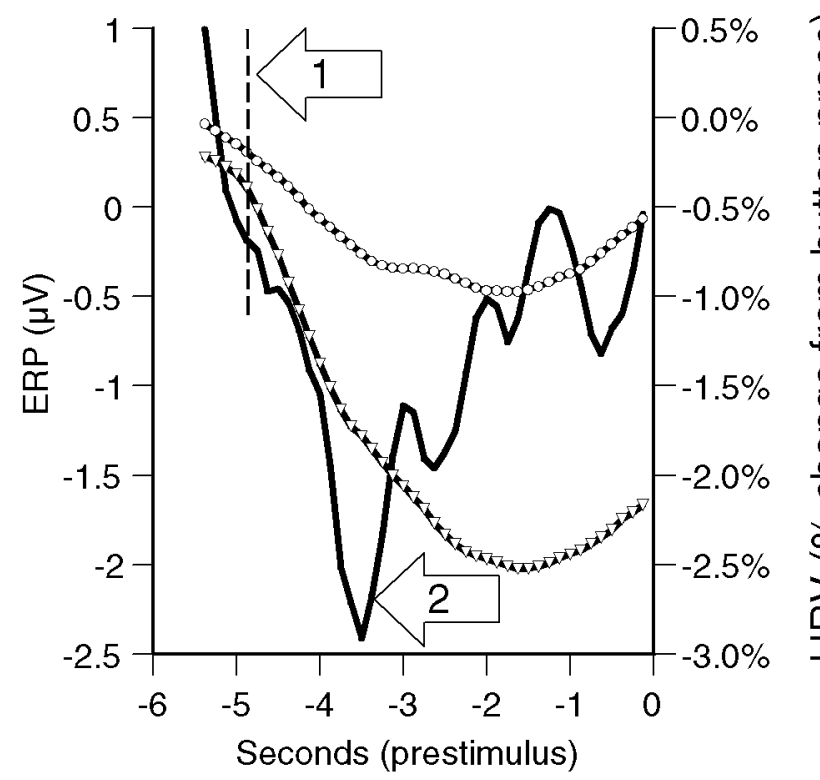

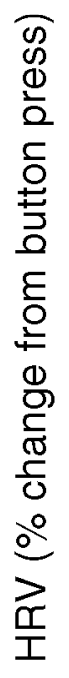

FIG. 5. Temporal dynamics of heart and brain prestimulus responses. This overlay plot shows the mean event-related potential (ERP) at FP2 and heart rate deceleration curves for the female subgroup $(n=15)$ in condition 1 during the prestimulus period. (The "0" time point denotes stimulus onset.) The heart rate deceleration curve for the emotional trials diverged from that of the calm trials (sharp downward shift) approximately 4.8 seconds prior to the stimulus (arrow 1), while the emotional trial ERP showed a sharp positive shift approximately 3.5 seconds prior to the stimulus (arrow 2). This positive shift in the ERP indicates when the brain "knew" the nature of the future stimulus. The time difference between these two events suggests that the heart received the intuitive information about 1.3 seconds before the brain.

discernible effects on perception. Moreover, cardiac afferent information has been demonstrated to interact with and modify the processing of sensory input (Walker and Sandman, 1979, 1982).

In condition 1 , the females had a significant $t_{\text {sum }}$ difference in the prestimulus HBEP in the right lateral frontal region (F8), with the potential for the emotional trials starting at a higher positive value than that for the calm trials. However, the emotional trial HBEP shifts sharply negative in closely coupled timing to the phase of the ECG where the voltage in the QRS complex is shifting negative. ${ }^{\dagger \dagger}$ In condition 2, the females had significant HBEP $t_{\text {sum }}$ differences in both frontopolar locations (FP1 and FP2), suggesting that when they maintained a state of physiological coherence

${ }^{\dagger \dagger \dagger}$ It should be noted that the HBEP waveforms are only $600 \mathrm{~ms}$ long and thus cannot be directly compared to the ERP waveforms, which are 6 seconds in duration. 
prior to the session, the cardiovascular-related signals were processed more frontally and in both hemispheres, rather than on the right side alone. These differences in HBEP waveforms in the prestimulus region between the calm and emotional trials suggest that afferent input to the brain from the heart contains information pertaining to the future stimulus.

\section{Intuition as a system-wide process}

This study has provided further evidence of what we are calling an intuitive effect. Although this phenomenon has previously been referred to as "precognition" and "presentiment," we question whether the former term is appropriate. This is because, in normal usage, "precognition" implies that cognition (knowing) is involved, whereas the evidence from electrophysiological studies of the prestimulus response suggest that the body's awareness of a future event is independent of conscious cognition (McDonough et al., 2002) $\$$ This does not mean, however, that cognition is not involved in the decoding and conscious perception of intuitive information as a feeling, image, auditory/linguistic translation, etc.

Overall, our data suggest that the apprehension of information pertaining to future emotional events is a systemwide process, rather than one that is localized to the brain alone. Moreover, our results provide evidence suggesting that the heart is involved and may play an important role in the processing of intuitive information. Figure 5 shows an overlay of the emotional trial ERPs at FP2 and the heart rate deceleration curves for the females in condition 1, which allows investigation of the sequence of changes in brain and heart activity indicative of a change in informational processing status. The heart rate deceleration curve in the emotional trials diverged from that of the calm trials approximately 4.8 seconds prior to the stimulus, while a sharp positive shift in the ERPs occurred approximately $3.5 \mathrm{sec}-$ onds prior to the stimulus. It would seem likely, therefore, that the heart received the intuitive information before the brain, as we argue below.

The deceleration in heart rate, indicating a shift in informational content, is usually interpreted as the result of an increase in parasympathetic outflow controlled solely by the brain. However, it is also possible that the deceleration originated within the heart itself, and that the resulting change in afferent neural signals to the brain either signaled the brain about the future event or facilitated its processing of the intuitive information, or both. This interpretation is consistent with the prestimulus HBEPs, which were significantly different in the emotional trials compared to the calm trials.

$\$+$ It is worth noting that an electophysiologica prestimulus response, which is an appropriate way of describing the physiologic data, has also been measured in earthworms, (see Wildey, 2001).
This possibility is also corroborated by recent research in neurocardiology, which has established that the heart is a sensory organ and an information encoding and processing center with an extensive intrinsic nervous system, enabling it to learn, remember, and make functional decisions independent of the cranial brain. The heart's intrinsic nervous system not only makes adjustments to the heart's rhythmic activity on a beat-to-beat basis but can even override inputs from the sympathetic and parasympathetic nervous systems (Armour, 2003; Armour and Ardell, 1994). Moreover, there is substantial evidence that patterns of cardiac afferent neurological input not only exert homeostatic effects on cardiovascular regulation via their interaction with brain stem autonomic regulatory centers, but also modulate the activity of subcortical and cortical regions of the brain involved in perceptual and emotional processing (Frysinger and Harper, 1990; McCraty and Childre, 2004; Sandman et al., 1982). Both neurophysiologic and behavioral data indicate that afferent input from the heart and cardiovascular system influences the processing of sensory information. For instance, studies have documented that cardiac-related afferent input modulates a wide range of processes such as visual perception (Walker and Sandman, 1982), reaction times (Lacey and Lacey, 1974), pain perception (Randich and Gebhart, 1992), electrocortical activity, and cognitive functions (Sandman et al., 1982; van der Molen et al., 1985; Rau et al., 1993). Given the complex, requisite contribution of input from the heart in influencing diverse aspects of sensory processing by the brain, it is reasonable to postulate that information from the heart may also inform intuitive perception.

\section{Correlates of intuition}

To our knowledge, this investigation makes a number of unique contributions to the study of intuition. One is that we measured the prestimulus response in two different psychophysiologic conditions: in a "baseline" psychophysiologic state; and after having maintained a state of physiological coherence. A second contribution is that we investigated electrophysiological prestimulus responses by gender. In Part 1 we reported that the females demonstrated a significant HRV response to the prestimulus information in condition 2 (physiological coherence), whereas the males did not (McCraty et al., 2004). Here in Part 2 we expanded the analysis to provide data on the brain's response to prestimulus information and data on the interactions between the heart and brain in processing intuitive information under the two experimental conditions and by gender.

The most interesting gender difference emerged from the ERP and HBEP data. In general, the females appear to process the prestimulus information more frontally (FP1 and FP2), while the males process it more in the posterior regions of the brain (T5, P3, O1, and $\mathrm{O} 2$ ).

For females, there were some other intriguing results. In 
condition 1, although the females' HBEPs were not significantly different in the frontal areas (FP1 and FP2), their emotional trial HBEP waveforms start at a more positive level and shift sharply negative. This negative shift in the HBEP waveforms adds to the overall negativity of the emotional trial ERPs, which were significantly more negative than the calm trial ERPs in condition 1.

In condition 2, however, a quite different picture emerges. In the coherent condition, the females' emotional trial ERPs did not have the increased negativity and were therefore not significantly different, but their HBEPs were significantly different. In this condition, the HBEPs start at a more negative level and shift sharply positive, apparently reducing the overall negativity of the ERPs (see Figs. 3 and 4). Thus, it would appear that afferent input from the heart plays an important role in modulating activity in the frontal cortex.

In short, taken as a whole, these findings suggest that females are more attuned to information from the heart, especially when the coherent mode is activated.

\section{Potential artifacts and limitations}

There are a number of sources of potential artifacts that we have examined and ruled out as an explanation for the intuitive effect observed here. These include: sensory or statistical cueing and participant anticipation effects; measurement, data collection, or data analysis artifacts; and participant or experimenter fraud. These and other factors have been examined in depth by other researchers (Bierman and Scholte, 2002; Radin, 1997b, 2004; Spottiswoode and May, 2003) and were carefully considered in the design and execution of our experiments. These potential sources of spuriousness were investigated and discounted as an explanation for the findings observed in this study (see Part 1 for a detailed discussion). Limitations of the study are also considered in Part 1 (McCraty et al., 2004).

\section{CONCLUSIONS}

We began our research with two empirical objectives. One was replication of the results of previous studies, which had documented a physiologic response to a future emotionally arousing stimulus before this stimulus was administered. Overall, our results were consistent with these studies, in that we found that certain electrophysiological indicators respond to a future emotionally arousing stimulus prior to individuals actually experiencing the future stimulus. This suggests that some aspect of our perceptual apparatus is continuously scanning the future, a point we expand on below.

The second objective was to extend the scope of prior studies and include measures of both brain and heart activity in order to better understand where and when in the body information about the future event is registered and processed. Our main findings were that: (1) a significant heart rate deceleration occurred prior to a future emotional stimulus (see Part 1); (2) there were significant differences in cortical ERPs, primarily in the frontal areas, with increased negativity and a faster onset of the positive-going wave in emotional trials; (3) the frontal cortex, left temporal area, occipital areas, and, to a lesser degree, parietal areas of the brain appear to be involved in the processing of intuitive information; (4) there were significant differences in heartbeat-evoked potentials between the calm and emotional trials; (5) there were significant physiologic differences associated with the two experimental conditions and gender in the processing of prestimulus information.

Of even greater significance, in relation to our second goal, is our major finding: namely, that both the heart and brain appear to receive and respond to information about a future emotional stimulus prior to actually experiencing the stimulus. The fact that the heart is involved in the perception of a future external event is a surprising, even astounding result, especially from the classical perspective that assigns the brain an exclusive role. Yet, along with other great thinkers, Einstein understood this long ago that " $[\mathrm{O}]$ nly intuition, resting on sympathetic understanding, can lead to [these laws]; ... the daily effort comes from no deliberate intention or program, but straight from the heart" (quoted in Keller, 1983).

In short, our findings suggest that intuitive perception is not a discrete function produced by a single part or system of the body alone. Rather, it appears that intuition may in fact be a system-wide process involving at least the heart and brain, together, in the processing and decoding of intuitive information.

These are quite amazing electrophysiologicalresults, which, if confirmed and extended by future research, will contribute to a new understanding of how the body receives and processes information about objects and events ahead in time or apart from the individual in space. But could they really be true? Because we have used rigorous, conservative methods, have ruled out the likely sources of spuriousness, and also have findings that are consistent with previous research, we expect our major results to be confirmed by other independent studies.

Assuming these psychophysiological effects continue to be demonstrated in future research, they are strong evidence for the idea that intuitive processes involve the body accessing a field of information that is not limited by the constraints of space and time. More specifically, they provide a compelling basis for the proposition that the body accesses a field of potential energy - that exists as a domain apart from space-time reality - into which information about "future" events is spectrally enfolded. This concept of the spectral enfoldment of information in the interaction among energy waveforms at "any" frequency (Gabor, 1946) provides an information processing bridge between the classical and quantum levels, and, therefore, informs the theory we develop in Part 3 of this work (forthcoming). Drawing on Pribram and Bradley's approach (Bradley, 1997; Bradley and Pribram, 1998; Pribram, 1991; Pribram and Bradley, 1998), 
we use this concept to show how the interaction between the body and the ambient field of energy surrounding it operates as a holographic-like information processing system that informs the body about a "future" event. $\$ \S \S$

The investigation of intuitive perception promises a rich harvest for humankind: an enlarged scientific understanding of human perception and consciousness, and even a new view of ourselves and of our relation to the material and nonmaterial worlds. In this rapidly changing and highly complex world of ours in the twenty-first century, we believe there is a critical role for intuitive perception in informing choices and decisions in virtually every aspect of human life. It is through scientific research, such as this, that we can build an understanding of how and under what conditions intuition occurs, and thus also learn the keys to harnessing and developing its power.

\section{ACKNOWLEDGMENTS}

The authors would like to acknowledge Karl Pribram, M.D., Ph.D., for his time and expertise in aiding in the appropriate interpretation of the ERP data. We also thank the anonymous reviewers of this article for their helpful suggestions, and are especially grateful to Dana Tomasino, B.A. (Hons.), whose tireless dedication resulted in significant substantive improvements to the quality and clarity of the manuscript. Finally, we thank the research participants, without whom this study would not have been possible.

\section{REFERENCES}

Aczel AD. Entanglement: The Greatest Mystery in Physics. New York: Four Walls Eight Windows, 2002.

Armour JA. Neurocardiology-Anatomical and functional principles. Boulder Creek, CA: HeartMath Research Center, Institute of HeartMath, Publication No. 03-011, 2003.

Armour JA, Ardell JL, eds. Neurocardiology. New York: Oxford University Press, 1994.

Ben-Jacob E, Cohen I, Levine H. Cooperative self-organization of microorganisms. Adv Phys 2000;49:395-554.

Bierman DJ. Anomalous baseline effects in mainstream emotion research using psychophysiological variables. Proceedings of presented papers at The 43rd Annual Convention of the Parapsychological Association, 2000:34-47.

Bierman DJ, Radin DI. Anomalous anticipatory response on randomized future conditions. Percept Mot Skills 1997;84:689-690.

$\S \S \S$ Holographic organization is based on a field concept of order, in which information about the organization of an object as a whole is encoded as an interference pattern in energy waveforms distributed throughout the field. This makes it possible to retrieve information about the object as a whole from any location within the field (Gabor, 1948).
Bierman DJ, Scholte HS. Anomalous anticipatory brain activation preceding exposure of emotional and neutral pictures. Paper presented at Toward a Science of Consciousness IV, Tuscon AZ, 2002.

Blair RC, Karniski W. An alternative method for significance testing of waveform difference potentials. Psychophysiology 1993; 30:518-524.

Bohm D. Wholeness and the Implicate Order. London: Routledge and Kegan Paul, 1980.

Bohm D, Hiley BJ. The Undivided Universe. London: Routledge, 1993.

Boucsein W. Electrodermal Activity. New York: Plenum Press, 1992.

Bradley RT. Charisma and Social Structure: A Study of Love and Power, Wholeness and Transformation. New York: Paragon House, 1987.

Bradley RT. The anticipation of order in biosocial collectives. World Futures 1997;49:65-88.

Bradley RT, Pribram KH. Communication and stability in social collectives. J Soc Evolutionary Systems 1998;21:29-80.

Childre D, Martin H. The HeartMath Solution. San Francisco: HarperSanFrancisco, 1999.

Don NS, McDonough BE, Warren CA. Event-related brain potential (ERP) indicators of unconsciouspsi: A replication using subjects unselected for psi. J Parapsychol 1998;62:127-145.

Frysinger RC, Harper RM. Cardiac and respiratory correlations with unit discharge in epileptic human temoral lobe. Epilepsia 1990;31:162-171.

Gabor D. Theory of communication. J Inst Electrical Engineers 1946;93:429-457.

Gabor D. A new microscopic principle. Nature 1948;161:777-778.

Hameroff SR. Ultimate Computing: Biomolecular Consciousness and Nanotechnology. Amsterdam: North Holland Press, 1987.

Keller EF. A Feeling for the Organism: The Life and Work of Barbara McClintock. New York: W.H. Freeman and Company, $1983 ; 201$ [quotes].

Lacey BC, Lacey JI. Studies of heart rate and other bodily processes in sensorimotor behavior. In: Obrist PA, Black AH, Brener J, DiCara LV, eds. Cardiovascular Psychophysiology: Current Issues in Response Mechanisms, Biofeedback, and Methodology. Chicago: Aldine, 1974:538-564.

Laszlo E. The Interconnected Universe: Conceptual Foundations of Transdisciplinary Unified Theory. Singapore: World Scientific, 1995.

Levin J, Kennedy J. The relationship of slow cortical potentials to psi information in man. J Parapsychol 1975;39:25-26.

Loye D. The Sphinx and the Rainbow: Brain, Mind and Future Vision. New York: Bantam Books, 1983.

McCraty R. Influence of cardiac afferent input on heart-brain synchronization and cognitive performance [abstract]. Int J Psychophysiol 2002;45:72-73.

McCraty R, Atkinson M. Psychophysiological coherence. Boulder Creek, CA: HeartMath Research Center, Institute of HeartMath, Publication 03-016, 2003.

McCraty R, Atkinson M, Bradley RT. Electrophysiological evidence of intuition: Part 1. The surprising role of the heart. J Altern Complement Med 2004;10:133-143.

McCraty R, Childre D. The appreciative heart: The psychophysiology of positive emotions and optimal functioning. Boulder Creek, CA: HeartMath Research Center, Institute of HeartMath, Publication No. 02-026, 2002. 
McCraty R, Childre D. The grateful heart: The psychophysiology of appreciation. In: Emmons RA, McCullough ME, eds. The Psychology of Gratitude. New York: Oxford University Press, 2004:230-255.

McDonough BE, Don NS, Warren CA. Differential event-related potentials to targets and decoys in a guessing task. J Sci Exploration 2002;16:187-206.

Myers DG. Intuition: Its Powers and Perils. New Haven: Yale University Press, 2002.

Naatanen R, Paavilainen P, Tiitinen H, Jiang D, Alho K. Attention and mismatch negativity. Psychophysiology 1993;30:436-450.

Nadeau R, Kafatos M. The Non-Local Universe: The New Physics and Matters of the Mind. New York: Oxford University Press, 1999.

Paige SR, Newton JE, Reese WG, Dykman RA. Pavlovian conditioning of cortical event-related potentials. In: Johnson R, Rohrbaugh JW, Parasuraman R, eds. Current Trends in EventRelated Potential Research, EEG Suppl. Amsterdam: Elsevier, 1987:355-359.

Penrose R. The Emperor's New Mind: Concerning Computers, Minds, and the Laws of Physics. New York: Oxford University Press, 1989.

Pribram KH. Brain and Perception: Holonomy and Structure in Figural Processing. Hillsdale, NJ: Lawrence Erlbaum Associates, Publishers, 1991.

Pribram KH, Bradley RT. The brain, the me and the I. In: Ferrari M, Sternberg R, eds. Self-Awareness: Its Nature and Development. New York: The Guilford Press, 1998:273-307.

Radin DI. The Conscious Universe. San Franscisco: HarperEdge, 1997a.

Radin DI. Unconscious perception of future emotions: An experiment in presentiment. J Sci Exploration 1997b;11:163-180.

Radin DI. Electrodermal presentiments of future emotions. J Sci Exploration 2004;18, in press.

Randich A, Gebhart GF. Vagal afferent modulation of nociception. Brain Res Rev 1992;17:77-99.

Rau H, Pauli P, Brody S, Elbert T. Baroreceptor stimulation alters cortical activity. Psychophysiol 1993;30:322-325.

Sandman CA, Walker BB, Berka C. Influence of afferent cardiovascular feedback on behavior and the cortical evoked potential. In: Cacioppo JT, Petty RE, eds. Perspectives in Cardiovascular Psychophysiology.New York: The Guilford Press, 1982:189-222. Schandry R, Montoya P. Event-related brain potentials and the processing of cardiac activity. Biol Psychol 1996;42:75-85.

Schandry R, Sparrer B, Weitkunat R. From the heart to the brain: A study of heartbeat contingent scalp potentials. Int J Neurosci 1986;30:261-275.
Sheldrake R. Dogs That Know When Their Owners Are Coming Home: And Other Unexplained Powers of Animals. New York: Three Rivers Press, 1999.

Sheldrake R, McKenna T, Abraham R. The Evolutionary Mind: Trialogues at the Edge of the Unthinkable. Santa Cruz, CA: Trialogue Press, 1998.

Shepherd GM, Brayton RK, Miller JP, Segev I, Rinzel J, Rall W. Signal enhancement in distal cortical dendrites by means of interactions between active dendritic spines. Proc Natl Acad Sci USA $1985 ; 82: 2192-2195$.

Spottiswoode J, May E. Skin conductance prestimulus response: Analyses, artifacts and a pilot study. J Sci Exploration 2003; 17:617-641.

Tiller WA, McCraty R, Atkinson M. Cardiac coherence: A new, noninvasive measure of autonomic nervous system order. Altern Ther Health Med 1996;2:52-65.

Tervaniemi M, Maury S, Naatanen R. Neural representations of abstract stimulus features in the human brain as reflected by the mismatch negativity. Neuroreport 1994;5:844-846.

van der Molen MW, Somsen RJM, Orlebeke JF. The rhythm of the heart beat in information processing. In: Ackles PK, Jennings JR, Coles MGH, eds. Advances in Psychophysiology, vol. 1. London: JAI Press, 1985:1-88.

Walker B, Sandman C. Visual evoked potentials change as heart rate and carotid pressure change. Psychophysiol 1982;19:520527.

Walker BB, Sandman CA. Human visual evoked responses are related to heart rate. J Comp Physiol Psychol 1979;93:18-25.

Warren CA, McDonough BE, Don NS. Event-related brain potential changes in a psi task. J Parapsychol 1992a;56:1-30.

Warren CA, McDonough BE, Don NS. Partial replication of single subject event-related potential effects in a psi task. The Parapsychological Association 35th Annual Convention: Proceedings of Presented Papers, 1992b:169-181.

Address reprint requests to: Rollin McCraty, Ph.D. HeartMath Research Center Institute of HeartMath 14700 West Park Avenue Boulder Creek, CA 95006

E-mail: rollin@heartmath.org 